\title{
Multiplicity results for an anisotropic equation with subcritical or critical growth
}

\author{
Giovany Figueiredo * and João R. Santos Junior ${ }^{\dagger}$ \\ Universidade Federal do Pará, \\ Faculdade de Matemática, \\ CEP: 66075-110 Belém - Pa , Brazil. \\ e-mail: giovany@ufpa.br and joaojunior@ufpa.br \\ and \\ Antonio Suarez $z^{\ddagger}$ \\ Dpto. de Ecuaciones Diferenciales y Análisis Numérico, \\ Fac. de Matemáticas, Univ. de Sevilla, \\ C/. Tarfia s/n, 41012 - Sevilla, SPAIN \\ e-mail: suarez.us.es
}

\begin{abstract}
In this work we show some multiplicity results for the anisotropic equation

$$
-\sum_{i=1}^{N} \frac{\partial}{\partial x_{i}}\left(\left|\frac{\partial u}{\partial x_{i}}\right|^{p_{i}-2} \frac{\partial u}{\partial x_{i}}\right)=g_{\lambda}(u) \text { in } \Omega, \text { and } u=0 \text { on } \partial \Omega
$$

where $\Omega \subset \mathbb{R}^{N}$ is a bounded smooth domain, $1<p_{1} \leq p_{2} \leq \ldots \leq p_{N}$ and $\lambda$ is a positive parameter. Using genus theory, we study the subcritical case $g_{\lambda}(u)=\lambda|u|^{q-2} u$ with $q \in\left(1, p_{N}\right)$ and the critical case $g_{\lambda}(u)=$ $\lambda|u|^{q-2} u+|u|^{p^{*}-2} u$ with $q \in\left(1, p_{1}\right)$ and $p^{*}=N \bar{p} /(N-\bar{p})$, with $\bar{p}$ the harmonic mean.
\end{abstract}

2000 Mathematics Subject Classification : 35J25, 35B65, 35J70 and $46 \mathrm{E} 35$.

Key words: Anisotropic operator, Genus theory, Subcritical or critical growth.

*Supported by PROCAD/CASADINHO: 552101/2011-7, CNPq/PQ 301242/2011-9 and CNPQ/CSF 200237/2012-8

$\dagger$ Supported by CAPES/PDSE - Brazil - 7155123/2012-9

${ }^{\ddagger}$ Supported by MICINN and FEDER under grant MTM 2012-31304 


\section{Introduction}

In this paper we are concerned with the multiplicity of nontrivial solutions for the following classes of nonlinear anisotropic problems

$\left(P 1_{\lambda}\right)$

$$
\left\{\begin{array}{l}
-\sum_{i=1}^{N} \frac{\partial}{\partial x_{i}}\left(\left|\frac{\partial u}{\partial x_{i}}\right|^{p_{i}-2} \frac{\partial u}{\partial x_{i}}\right)=\lambda|u|^{q-2} u \text { in } \Omega \\
u \in D_{0}^{1, \vec{p}}(\Omega), \quad q \in\left(1, p_{N}\right)
\end{array}\right.
$$

and

$$
\left\{\begin{array}{l}
-\sum_{i=1}^{N} \frac{\partial}{\partial x_{i}}\left(\left|\frac{\partial u}{\partial x_{i}}\right|^{p_{i}-2} \frac{\partial u}{\partial x_{i}}\right)=\lambda|u|^{q-2} u+|u|^{p^{*}-2} u \text { in } \Omega \\
u \in D_{0}^{1, \vec{p}}(\Omega), \quad q \in\left(1, p_{1}\right),
\end{array}\right.
$$

where $\Omega$ is a bounded smooth domain in $\mathbb{R}^{N}, N \geq 3, \lambda$ is a positive parameter,

$$
\begin{gathered}
1<p_{1} \leq p_{2} \leq \ldots \leq p_{N}, \quad \sum_{i=1}^{N} \frac{1}{p_{i}}>1, \\
D_{0}^{1, \vec{p}}(\Omega):=\left\{u \in L^{p^{*}}(\Omega): \frac{\partial u}{\partial x_{i}} \in L^{p_{i}}(\Omega) ; i=1, \ldots, N\right\},
\end{gathered}
$$

$\vec{p}=\left(p_{1}, \ldots, p_{N}\right)$, and

$$
p^{*}:=\frac{N}{\left(\sum_{i=1}^{N} \frac{1}{p_{i}}\right)-1}=\frac{N \bar{p}}{N-\bar{p}}
$$

where $\bar{p}$ denotes the harmonic mean $\bar{p}=N /\left(\sum_{i=1}^{N} \frac{1}{p_{i}}\right)$.

Throughout all the paper, we assume that

$$
p_{N}<p^{*}
$$

Observe that the anisotropic operator is a generalization of the Laplacian one. Indeed, when $p_{i}=2$ for all $i=1, \ldots, N$, then

$$
\sum_{i=1}^{N} \frac{\partial}{\partial x_{i}}\left(\left|\frac{\partial u}{\partial x_{i}}\right|^{p_{i}-2} \frac{\partial u}{\partial x_{i}}\right)=\Delta u .
$$


A considerable effort has been devoted during the last years to the study anisotropic problems. With no hope to be thorough, let us mention, for example, [1], [9], [10], [11], [15], [16], [17], [20], [21], [22], [23], [24], [26], [27] and references given there.

This is greatly justified in view of two basic aspects of mathematical research. The first one is that this class of problems has a rich physical motivation. It appears, for instance, in biology, see [7] and [8], as a model describing the spread of an epidemic disease in heterogeneous environments. It also emerges, see [3] and [5], from the mathematical description of the dynamics of fluids with different conductivities in different directions. To application in image processing, see [25].

The second aspect of the relevance of anisotropic problems is related to the mathematical techniques used to approach it. Sometimes, some refined estimates are needed due to different orders of derivation of the operator in different directions.

In this paper we are interested in giving some multiplicity results, which complete the existing in the literature. With respect to $\left(P 1_{\lambda}\right)$, the main results are:

Theorem 1.1. Assume that $q \in\left(1, p_{1}\right)$. Then, problem $\left(P 1_{\lambda}\right)$ has infinitely many solutions, for all $\lambda \in(0,+\infty)$.

Theorem 1.2. Assume that $q \in\left[p_{1}, p_{N}\right)$. Then, for each $k \in \mathbb{N}$, there exists $\lambda_{k}>0$ such that problem $\left(P 1_{\lambda}\right)$ has at least $k$ pairs of solutions, for all $\lambda \in\left(\lambda_{k},+\infty\right)$.

With respect to $\left(P 2_{\lambda}\right)$ we have:

Theorem 1.3. Assume that $q \in\left(1, p_{1}\right)$. Then, there exists $\lambda^{*}>0$ such that problem $\left(P 2_{\lambda}\right)$ has infinitely many solutions, for all $\lambda \in\left(0, \lambda^{*}\right)$.

In some sense our paper is a natural continuation of the studies initiated in [1], [11] and [17] and it completes the results obtained there. Indeed, in [17], the authors studied important properties on the Banach space $D_{0}^{1, \vec{p}}(\Omega)$ and they showed that problem $\left(P 1_{\lambda}\right)$ with $q \in\left(p_{N}, p^{*}\right)$ has one solution for all $\lambda>0$.

For the case $q \in\left(p_{1}, p_{N}\right)$, it was proved in [11] that problem $\left(P 1_{\lambda}\right)$ possesses at least one solution for large $\lambda$ and no solution when $\lambda$ is small.

In [1], the authors showed that problem $\left(P 2_{\lambda}\right)$ has one solution when $q \in\left(1, p_{1}\right)$ and $\lambda$ small and when $q \in\left(p_{N}, p^{*}\right)$ and $\lambda$ is large.

In order to prove our results, mainly we have used variational methods. Thus, for Theorems 1.1 and 1.2 we utilize notions on the Krasnoleskii genus and Clarke's Theorem. The proof of the Theorem 1.3 is more complicated. We have used a similar idea to that of [4], where the authors showed a multiplicity result for problem

$$
\left\{\begin{array}{l}
-\Delta_{p} u=\lambda|u|^{q-2} u+|u|^{p^{*}-2} u \text { in } \Omega, \\
u \in W_{0}^{1, p}(\Omega), \quad q \in(1, p),
\end{array}\right.
$$


see also [14], where a nonlocal operator is considered. However, due to the anisotropic operator, we need to prove new bounds for the truncated functional, see Section 5 for details.

The plan of this paper is as follows. In Section 2, we write our problem in a variational framework. In Section 3, we recall some properties of genus theory and Clarke's Theorem. We study in Section 4 the subcritical case. Finally, in the Section 5, we analyze the critical case.

\section{Variational framework}

It is well known that $D_{0}^{1, \vec{p}}(\Omega)$, which is the completion of the space $\mathcal{D}(\Omega)$ with respect to the norm

$$
\|u\|=\sum_{i=1}^{N}\left|\frac{\partial u}{\partial x_{i}}\right|_{p_{i}}
$$

is a reflexive Banach space and is continuously embedded in $L^{p^{*}}(\Omega)$. Here $|\cdot|_{p_{i}}$ is the usual norm in $L^{p_{i}}(\Omega)$.

Since $\Omega$ is a bounded domain of $\mathbb{R}^{N}$, from [17, Theorem 1], the continuity of the embedding $D_{0}^{1, \vec{p}}(\Omega) \hookrightarrow L^{s}(\Omega)$, for all $s \in\left[1, p^{*}\right]$ relies on a well-known Poincaré-type inequality. More precisely, denoting by $e_{1}, \ldots, e_{n}$ the canonical basis of $\mathbb{R}^{N}$, assume that $\Omega$ has width $a>0$ in the direction of $e_{i}$, namely $\sup _{x, y \in \Omega}\left(x-y, e_{i}\right)=a$. Thus, for every $q \geq 1$, we have

$$
|u|_{q} \leq \frac{a q}{2}\left|\frac{\partial u}{\partial x_{i}}\right|_{q}, \quad \text { for all } u \in \mathcal{D}(\Omega)
$$

Definition 2.1. We say that $u \in D_{0}^{1, \vec{p}}(\Omega)$ is a weak solution of the problem $\left(P i_{\lambda}\right), i=1,2$ if it verifies

$$
\sum_{i=1}^{N} \int_{\Omega}\left|\frac{\partial u}{\partial x_{i}}\right|^{p_{i}-2} \frac{\partial u}{\partial x_{i}} \frac{\partial \phi}{\partial x_{i}} d x-\lambda \int_{\Omega}|u|^{q-2} u \phi d x-\int_{\Omega} h(u) \phi d x=0
$$

for all $\phi \in D_{0}^{1, \vec{p}}(\Omega)$, where $h(t)=0$ in problem $\left(P 1_{\lambda}\right)$ and $h(t)=|t|^{2^{*}-2} t$ in problem $\left(P 2_{\lambda}\right)$.

If a function $u \in D_{0}^{1, \vec{p}}(\Omega) \bigcap L^{\infty}(\Omega)$ satisfies (2.2), then $u$ is a strong solution of the problem $\left(P i_{\lambda}\right)$. From [1, Lemma 4.1] and [17, Theorem 4], weak solutions of problem $\left(P i_{\lambda}\right), i=1,2$ are strong solutions.

We will look for solutions of $\left(P i_{\lambda}\right), i=1,2$ by finding critical points of the $C^{1}$-functional $I: D_{0}^{1, \vec{p}}(\Omega) \rightarrow \mathbb{R}$ given by

$$
I(u)=\sum_{i=1}^{N} \int_{\Omega} \frac{1}{p_{i}}\left|\frac{\partial u}{\partial x_{i}}\right|^{p_{i}} d x-\lambda \frac{1}{q} \int_{\Omega}|u|^{q} d x-\int_{\Omega} H(u) d x,
$$


only in the case $h(t)=0$ and $h(t)=|t|^{2^{*}-2} t$, where $H(t)=\int_{0}^{t} h(\tau) d \tau$.

Note that

$$
I^{\prime}(u) \phi=\sum_{i=1}^{N} \int_{\Omega}\left|\frac{\partial u}{\partial x_{i}}\right|^{p_{i}-2} \frac{\partial u}{\partial x_{i}} \frac{\partial \phi}{\partial x_{i}} d x-\lambda \int_{\Omega}|u|^{q-2} u \phi d x-\int_{\Omega} h(u) \phi d x,
$$

for all $\phi \in D_{0}^{1, \vec{p}}(\Omega)$. Hence critical points of $I$ are weak solutions for $\left(P i_{\lambda}\right), i=1,2$.

In order to use variational methods, we first derive some results related to the Palais-Smale compactness condition.

We say that a sequence $\left(u_{n}\right) \subset D_{0}^{1, \vec{p}}(\Omega)$ is a Palais-Smale sequence for the functional $I$ if

$$
I\left(u_{n}\right) \rightarrow c_{*} \text { and }\left\|I^{\prime}\left(u_{n}\right)\right\| \rightarrow 0 \text { in }\left(D_{0}^{1, \vec{p}}(\Omega)\right)^{\prime},
$$

for some $c_{*} \in \mathbb{R}$.

If (2.3) implies the existence of a subsequence $\left(u_{n_{j}}\right) \subset\left(u_{n}\right)$ which converges in $D_{0}^{1, \vec{p}}(\Omega)$, we say that $I$ satisfies the Palais-Smale condition. If this strongly convergent subsequence exists only for some $d$ values, we say that $I$ verifies a local Palais-Smale condition.

\section{Abstract results}

We will start by considering some basic notions on the Krasnoselskii genus which we will use in the proof of our main results.

Let $E$ be a real Banach space. Let us denote by $\mathfrak{A}$ the class of all closed subsets $A \subset E \backslash\{0\}$ that are symmetric with respect to the origin, that is, $u \in A$ implies $-u \in A$.

Definition 3.1. Let $A \in \mathfrak{A}$. The Krasnoselskii genus $\gamma(A)$ of $A$ is defined as being the least positive integer $k$ such that there is an odd mapping $\phi \in C\left(A, \mathbb{R}^{k}\right)$ such that $\phi(x) \neq 0$ for all $x \in A$. If $k$ does not exist we set $\gamma(A)=\infty$. Furthermore, by definition, $\gamma(\emptyset)=0$.

In the sequel we will establish only the properties of the genus that will be used through this work. More information on this subject may be found in the references [2], [12], [13] and [18].

Proposition 3.2. Let $A$ and $B$ be sets in $\mathfrak{A}$.

(i) If there exists an odd application $\varphi \in C(A, B)$ then $\gamma(A) \leq \gamma(B)$.

(ii) If there exists an odd homeomorphism $\varphi: A \rightarrow B$ then $\gamma(A)=\gamma(B)$.

(iii) If $A$ is a compact set, then there exists a neighborhood $K \in \mathfrak{A}$ of $A$ such that $\gamma(A)=\gamma(K)$.

(iv) If $\gamma(B)<\infty$, then $\gamma(\overline{A \backslash B}) \geq \gamma(A)-\gamma(B)$.

(v) If $\gamma(A) \geq 2$, then $A$ has infinitely many points. 
Proposition 3.3. Let $E=\mathbb{R}^{N}$ and $\partial \Omega$ be the boundary of an open, symmetric and bounded subset $\Omega \subset \mathbb{R}^{N}$ with $0 \in \Omega$. Then $\gamma(\partial \Omega)=N$.

Corollary 3.4. $\gamma\left(\mathcal{S}^{N-1}\right)=N$ where $\mathcal{S}^{N-1}$ is a unit sphere of $\mathbb{R}^{N}$.

We now establish a result due to Clarke [19].

Theorem 3.5. Let $J \in C^{1}(X, \mathbb{R})$ be a functional satisfying the Palais-Smale condition. Furthermore, let us suppose that

$\left.A_{1}\right) J$ is bounded from below and even;

$\left.A_{2}\right)$ there is a compact set $K \in \mathfrak{A}$ such that $\gamma(K)=k$ and $\sup _{x \in K} J(x)<J(0)$.

Then $J$ possesses at least $k$ pairs of distinct critical points and their corresponding critical values $c_{j}$ are less than $J(0)$.

\section{Subcritical case}

In this section we study some properties related to the functional $I: D_{0}^{1, \vec{p}}(\Omega) \rightarrow \mathbb{R}$, given by

$$
I(u)=\sum_{i=1}^{N} \int_{\Omega} \frac{1}{p_{i}}\left|\frac{\partial u}{\partial x_{i}}\right|^{p_{i}} d x-\lambda \frac{1}{q} \int_{\Omega}|u|^{q} d x .
$$

The next two lemmas are true for $q \in\left(1, p_{N}\right)$. In [11] the authors showed that $I$ is coercive when $q \in\left(p_{1}, p_{N}\right)$, by using the boundedness of levels sets $I^{b}=\left\{u \in D_{0}^{1, \vec{p}}(\Omega): I(u) \leq b\right\}$. In the following lemma we will show this same fact for $q \in\left(1, p_{N}\right)$ with simpler arguments.

Lemma 4.1. I is bounded from below.

Proof. We will show that $I$ is coercive. In fact, suppose by contradiction that $\|u\| \rightarrow \infty$. Unfortunately, we can not to assure that $\left|\frac{\partial u}{\partial x_{i}}\right|_{p_{i}} \rightarrow \infty$ for all $i \in$ $\{1, \ldots, N\}$. Hence, we will consider two cases.

If $|u|_{q}$ is bounded, then we have already $I(u) \rightarrow \infty$. On the other hand, if $|u|_{q} \rightarrow \infty$ then, by using Holder's inequality and (2.1), we conclude that

$$
\left|\frac{\partial u}{\partial x_{i}}\right|_{p_{i}} \rightarrow \infty, q \leq p_{i}
$$

Moreover, for some $q<p_{i}$ fixed, we have

$$
I(u) \geq \frac{1}{p_{i}}\left|\frac{\partial u}{\partial x_{i}}\right|_{p_{i}}^{p_{i}}-\frac{C}{q} \lambda\left|\frac{\partial u}{\partial x_{i}}\right|_{p_{i}}^{q} d x .
$$

It follows from (4.1) that $I(u) \rightarrow \infty$. In any case, $I$ is coercive and, therefore, $I$ is bounded from below. 
Lemma 4.2. I satisfies the $(P S)$ condition.

Proof. Let $\left(u_{n}\right)$ be a sequence in $D_{0}^{1, \vec{p}}(\Omega)$ such that

$$
I\left(u_{n}\right) \rightarrow C \text { and } I^{\prime}\left(u_{n}\right) \rightarrow 0 .
$$

Since $I$ is coercive, we conclude that $\left(u_{n}\right)$ is bounded in $D_{0}^{1, \vec{p}}(\Omega)$. Thus, passing to a subsequence, if necessary, we have

$$
\begin{gathered}
u_{n} \rightarrow u \text { in } D_{0}^{1, \vec{p}}(\Omega), \\
u_{n} \rightarrow u \text { in } L^{\sigma}(\Omega) \text { with } \sigma \in\left[1, p^{*}\right),
\end{gathered}
$$

and

$$
u_{n}(x) \rightarrow u(x) \text { a.e in } \Omega \text {. }
$$

Thus, from convergence in $L^{\sigma}(\Omega)$ we get

$$
\int_{\Omega}\left|u_{n}\right|^{q} d x-\int_{\Omega}\left|u_{n}\right|^{q-2} u_{n} u d x=o_{n}(1),
$$

and from weak convergence

$$
\sum_{i=1}^{N} \int_{\Omega}\left|\frac{\partial u}{\partial x_{i}}\right|^{p_{i}-2} \frac{\partial u}{\partial x_{i}} \frac{\partial u_{n}}{\partial x_{i}} d x-\sum_{i=1}^{N} \int_{\Omega}\left|\frac{\partial u}{\partial x_{i}}\right|^{p_{i}} d x=o_{n}(1) .
$$

Hence, from (4.3) we obtain

$$
\begin{aligned}
0 & \leq C_{p} \sum_{i=1}^{N}\left|\frac{\partial u_{n}}{\partial x_{i}}-\frac{\partial u}{\partial x_{i}}\right|_{p_{i}}^{p_{i}} \\
& \leq \sum_{i=1}^{N} \int_{\Omega}\left(\left|\frac{\partial u_{n}}{\partial x_{i}}\right|^{p_{i}-2} \frac{\partial u_{n}}{\partial x_{i}}-\left|\frac{\partial u}{\partial x_{i}}\right|^{p_{i}-2} \frac{\partial u}{\partial x_{i}}\right)\left(\frac{\partial u_{n}}{\partial x_{i}}-\frac{\partial u}{\partial x_{i}}\right) d x \\
& =\sum_{i=1}^{N} \int_{\Omega}\left|\frac{\partial u_{n}}{\partial x_{i}}\right|^{p_{i}} d x-\sum_{i=1}^{N} \int_{\Omega}\left|\frac{\partial u_{n}}{\partial x_{i}}\right|^{p_{i}-2} \frac{\partial u_{n}}{\partial x_{i}} \frac{\partial u}{\partial x_{i}} d x+o_{n}(1) .
\end{aligned}
$$

From (4.2), we derive

$$
0 \leq C_{p} \sum_{i=1}^{N}\left|\frac{\partial u_{n}}{\partial x_{i}}-\frac{\partial u}{\partial x_{i}}\right|_{p_{i}}^{p_{i}} \leq I^{\prime}\left(u_{n}\right) u_{n}-I^{\prime}\left(u_{n}\right) u+o_{n}(1),
$$

where $C_{p}$ is a constant which appears in the standard inequality in $\mathbb{R}$ given by

$$
\left(|x|^{p-2} x-|y|^{p-2} y\right)(x-y) \geq C_{p}|x-y|^{p},
$$


if $p \geq 2$ or

$$
\left(|x|^{p-2} x-|y|^{p-2} y\right)(x-y) \geq \frac{C_{p}|x-y|^{2}}{(|x|+|y|)^{2-p}},
$$

if $1<p<2$.

Thus, we conclude that $u_{n} \rightarrow u$ in $D_{0}^{1, \vec{p}}(\Omega)$ and the proof is complete.

\subsection{Proof of Theorem 1.1}

Let $X_{k}=\operatorname{span}\left\{e_{1}, e_{2}, \ldots, e_{k}\right\}$ be a subspace of $D_{0}^{1, \vec{p}}(\Omega)$ with $\operatorname{dim} X_{k}=k$. Note that $X_{k}$ is continuously embedded in $L^{q}(\Omega)$. Thus, the norms of $D_{0}^{1, \vec{p}}(\Omega)$ and $L^{q}(\Omega)$ are equivalent on $X_{k}$ and there exists a positive constant $C(k)$ which depends on $k$, such that

$$
-C(k)\|u\|^{q} \geq-\int_{\Omega}|u|^{q} d x, \text { for all } u \in X_{k} .
$$

Thus we conclude that

$$
I(u) \leq \sum_{i=1}^{N} \frac{1}{p_{i}}\left|\frac{\partial u}{\partial x_{i}}\right|_{p_{i}}^{p_{i}}-\lambda C(k) \frac{1}{q}\|u\|^{q} .
$$

Let $0<R<1$ and $u \in D_{0}^{1, \vec{p}}(\Omega)$ be such that $\|u\| \leq R$. Thus

$$
I(u) \leq \frac{1}{p_{1}}\|u\|^{p_{1}}-\lambda C(k) \frac{1}{q}\|u\|^{q}=\|u\|^{q}\left[\frac{1}{p_{1}}\|u\|^{p_{1}-q}-\lambda C(k) \frac{1}{q}\right] .
$$

Choosing $R<\min \left\{1, \lambda\left(\frac{C(k) p_{1}}{q}\right)^{\frac{1}{p_{1}-q}}\right\}$ we have

$$
I(u)<R^{q}\left[\frac{1}{p_{1}} R^{p_{1}-q}-\lambda C(k) \frac{1}{q}\right]<0=I(0),
$$

for all $u \in K=\left\{u \in X_{k}:\|u\|=R\right\}$. This inequality implies

$$
\sup _{u \in K} I(u)<0=I(0) .
$$

Since $X_{k}$ and $\mathbb{R}^{k}$ are isomorphic and $K$ and $\mathcal{S}^{k-1}$ are homeomorphic, we conclude that $\gamma(k)=k$. Moreover, $I$ is even. By Clarke's theorem (Theorem 3.5), $I$ has at least $k$ pairs of different critical points. Since $k$ is arbitrary, we found infinitely many critical points of $I$. 


\subsection{Proof of Theorem 1.2}

Before of the proof, we will need the following lemma.

Lemma 4.3. Let $A$ be the set defined by

$$
A=\left\{u \in D_{0}^{1, \vec{p}}(\Omega) \backslash\{0\}:\left|\frac{\partial u}{\partial x_{i}}\right|_{p_{i}}^{p_{i}} \leq\left|\frac{\partial u}{\partial x_{N}}\right|_{p_{N}}^{p_{N}}, i=1, \ldots, N-1\right\} .
$$

For each compact set $K \subset D_{0}^{1, \vec{p}}(\Omega) \backslash\{0\}$, there exists $t_{K}>0$ such that $t K \subset A$ for all $t \geq t_{K}$, where $t K=\{t u: u \in K\}$.

Proof. By using (2.1), we define the continuous functions $h_{i}: D_{0}^{1, \vec{p}}(\Omega) \backslash\{0\} \rightarrow \mathbb{R}$ by

$$
h_{i}(u)=\frac{\left|\frac{\partial u}{\partial x_{i}}\right|_{p_{i}}^{p_{i}}}{\left|\frac{\partial u}{\partial x_{N}}\right|_{p_{N}}^{p_{N}}}, \quad i \in\{1, \ldots, N-1\} .
$$

Since $K$ is compact, there exists $u_{i} \in K$ such that $h_{i}(u) \leq h_{i}\left(u_{i}\right)$ for all $u \in K$. Define still $t_{i}:=\left[h_{i}\left(u_{i}\right)\right]^{\frac{1}{p_{N}-p_{i}}}, h_{j}\left(u_{j}\right):=\max _{1 \leq i \leq N-1} h_{i}\left(u_{i}\right)$ and choose $t_{K}=t_{j}$. Thus, if $t \geq t_{K}$ we have $t \geq t_{i}$ and $t^{p_{N}-p_{i}} \geq t_{i}^{p_{N}-p_{i}}=h_{i}\left(u_{i}\right)$. Consequently,

$$
\frac{\left|\frac{\partial u}{\partial x_{i}}\right|_{p_{i}}^{p_{i}} \leq t^{p_{N}-p_{i}}}{\left|\frac{\partial u}{\partial x_{N}}\right|_{p_{N}}^{p_{N}}}
$$

and

$$
\left|\frac{\partial(t u)}{\partial x_{i}}\right|_{p_{i}}^{p_{i}} \leq\left|\frac{\partial(t u)}{\partial x_{N}}\right|_{p_{N}}^{p_{N}}, \forall u \in K \text { and } \forall i \in\{1, \ldots, N-1\} .
$$

Finally, we are ready to prove Theorem 1.2 .

Proof of Theorem 1.2: In a similar way to the previous theorem, for each $k \in \mathbb{N}$, we consider a k-dimensional subspace $X_{k}=\operatorname{span}\left\{e_{1}, e_{2}, \ldots, e_{k}\right\}$ of $D_{0}^{1, \vec{p}}(\Omega)$, continuously embedded in $L^{p_{N}}(\Omega)$. This is, there exists a positive constant $C(k)$ which depends on $k$, such that

$$
-C(k)\left|\frac{\partial u}{\partial x_{N}}\right|_{p_{N}} \geq-|u|_{p_{N}}, \text { for all } u \in X_{k} .
$$

Denoting by $S_{k}$ the unit sphere of $X_{k}$ and noting that $S_{k} \subset D_{0}^{1, \vec{p}}(\Omega) \backslash\{0\}$ is a compact set, it follows from previous lemma that there exists $t_{k}>0$ such that $t S_{k} \subset A$, for all $t \geq t_{k}$. Thus, for each $u \in t_{k} S_{k}$, we have

$$
I(u) \leq \frac{N}{\bar{p}}\left|\frac{\partial u}{\partial x_{N}}\right|_{p_{N}}^{p_{N}}-\frac{C(k)}{q} \lambda\left|\frac{\partial u}{\partial x_{N}}\right|_{p_{N}}^{q},
$$


and so

$$
I(u) \leq\left|\frac{\partial u}{\partial x_{N}}\right|_{p_{N}}^{q}\left(\frac{N}{\bar{p}}\left|\frac{\partial u}{\partial x_{N}}\right|_{p_{N}}^{p_{N}-q}-\frac{C(k)}{q} \lambda\right) .
$$

From (2.1) we conclude that $\alpha:=\min _{u \in t_{k} S_{k}}\left|\frac{\partial u}{\partial x_{N}}\right|_{p_{N}}>0$. Hence,

$$
I(u) \leq \alpha^{q}\left(\frac{N}{\bar{p}} t_{k}^{p_{N}-q}-\frac{C(k)}{q} \lambda\right)<0,
$$

when $\lambda>\lambda_{k}=\frac{q N}{\bar{p} C(k)} t_{k}^{p_{N}-q}$. Therefore,

$$
\sup _{t_{k} S_{k}} I_{\lambda}<0, \forall \lambda \geq \lambda_{k}
$$

with $\gamma\left(t_{k} S_{k}\right)=k$. Arguing as in the proof of Theorem 1.1, the result follows from Clarke's Theorem (Theorem 3.5).

\section{Critical case}

Since $I$ is not bounded from below, in the critical case, to apply genus theory, we will need to make a truncation in the functional $I$. In fact, the idea is to get a truncated functional $J$ such that critical points $u$ of $J$ with $J(u)<0$ are also critical points of $I$.

However, the anisotropy of $\left(P 2_{\lambda}\right)$ becomes our job somewhat more complicated. To overcome the difficulties, we need to consider separately the cases $\|u\| \leq 1$ and $\|u\| \geq 1$ in the building of $J$.

Case 1: $\|u\| \leq 1$.

In this case, we have $\left|\frac{\partial u}{\partial x_{i}}\right|_{p_{i}} \leq 1$ for all $i \in\{1, \ldots, N\}$, and consequently

$$
\left|\frac{\partial u}{\partial x_{i}}\right|_{p_{i}}^{p_{N}} \leq\left|\frac{\partial u}{\partial x_{i}}\right|_{p_{i}}^{p_{i}}
$$

Hence

$$
I(u) \geq \frac{1}{p_{N}} \sum_{i=1}^{N}\left|\frac{\partial u}{\partial x_{i}}\right|_{p i}^{p_{N}}-\lambda \frac{1}{q} \int_{\Omega}|u|^{q} d x-\frac{1}{p^{*}} \int_{\Omega}|u|^{p^{*}} d x .
$$

From continuous embedding,

$$
\int_{\Omega}|u|^{s} d x \leq C\|u\|^{s}, \quad s \in\left[1, p^{*}\right] .
$$


From previous inequality we obtain

$$
I(u) \geq \frac{C_{1}}{p_{N}}\|u\|^{p_{N}}-\lambda C\|u\|^{q}-C_{2}\|u\|^{p^{*}} \geq g(\|u\|),
$$

where $g(t)=\frac{C_{1}}{p_{N}} t^{p_{N}}-\lambda C t^{q}-C_{2} t^{p^{*}}$.

So, there exists $\lambda^{*}>0$ such that, if $\lambda \in\left(0, \lambda^{*}\right)$, then $g$ attains its positive maximum.

We denote by $0<R_{0}(\lambda)<R_{1}(\lambda)$ the unique two roots of $g$. The next lemma is essential to construct the truncated functional.

Lemma 5.1. $R_{0}(\lambda) \rightarrow 0$ as $\lambda \rightarrow 0$.

Proof. Indeed, from $g\left(R_{0}(\lambda)\right)=0$ and $g^{\prime}\left(R_{0}(\lambda)\right)>0$, we have

$$
\frac{C_{1}}{p_{N}} R_{0}(\lambda)^{p_{N}}=\lambda C R_{0}(\lambda)^{q}+C_{2} R_{0}(\lambda)^{p^{*}}
$$

and

$$
C_{1} R_{0}(\lambda)^{p_{N}-1}>\lambda C q R_{0}(\lambda)^{q-1}+C_{2} p^{*} R_{0}(\lambda)^{p^{*}-1},
$$

for all $\lambda \in\left(0, \lambda^{*}\right)$. From (5.2), we conclude that $R_{0}(\lambda)$ is bounded. Suppose that $R_{0}(\lambda) \rightarrow R_{0}>0$ as $\lambda \rightarrow 0$. Then,

$$
\frac{C_{1}}{p_{N}} R_{0}^{p_{N}}=C_{2} R_{0}^{p^{*}} \text { and } C_{1} R_{0}^{p_{N}-1} \geq C_{2} p^{*} R_{0}^{p^{*}-1}
$$

a contradiction, because $p^{*}>p_{N}$. Therefore $R_{0}=0$.

Now we consider the following truncation in the functional $I$ :

From Lemma 5.1, we have $R_{0}(\lambda)<1$ for small $\lambda$. So $R_{0}(\lambda)<\min \left\{R_{1}(\lambda), 1\right\}$ and we can take $\phi \in C_{0}^{\infty}([0,+\infty)), 0 \leq \phi(t) \leq 1$, for all $t \in[0,+\infty)$, such that

$$
\phi(t)=\left\{\begin{array}{l}
1, t \in\left[0, R_{0}(\lambda)\right] \\
0, t \in\left[\min \left\{R_{1}(\lambda), 1\right\},+\infty\right) .
\end{array}\right.
$$

We define the functional

$$
J(u)=\sum_{i=1}^{N} \int_{\Omega} \frac{1}{p_{i}}\left|\frac{\partial u}{\partial x_{i}}\right|^{p_{i}} d x-\lambda \frac{1}{q} \int_{\Omega}|u|^{q} d x-\phi(\|u\|) \frac{1}{p^{*}} \int_{\Omega}|u|^{p^{*}} d x .
$$

Note that $J \in C^{1}\left(D_{0}^{1, \vec{p}}(\Omega), \mathbb{R}\right)$ and, as in $(5.1), J(u) \geq \bar{g}(\|u\|)$, for all $u \in D_{0}^{1, \vec{p}}(\Omega)$ with $\|u\|<1$, where

$$
\bar{g}(t)=\frac{C_{1}}{p_{N}} t^{p_{i}}-\lambda C t^{q}-C_{2} \phi(t) t^{p^{*}} \geq 0, \forall t \in\left(R_{0}(\lambda), \min \left[R_{1}(\lambda), 1\right\}\right] .
$$


By definition, if $\|u\| \leq R_{0}(\lambda)<\min \left\{R_{1}(\lambda), 1\right\}$ then $J(u)=I(u)$. Once we will obtain critical points $u$ of $J$ with $J(u)<0$, to show that these critical points verify $\|u\|<R_{0}(\lambda)$ is important to ensure that $J(u) \geq 0$ when $\|u\|>1$.

In fact, suppose just for a moment that $J(u) \geq 0$ when $\|u\|>1$. Let $\bar{u}$ be a critical point of $J$ such that

$$
J(\bar{u})<0 .
$$

So $\|\bar{u}\| \leq 1$. If $\min \left\{R_{1}(\lambda), 1\right\}=1$, follows from (5.4) and (5.5) that $\|\bar{u}\|<R_{0}(\lambda)$. On the other hand, if $\min \left\{R_{1}(\lambda), 1\right\}=R_{1}(\lambda)$, we conclude again from (5.4), (5.5) and definition of $J$ that $\|\bar{u}\|<R_{0}(\lambda)$. It remains to prove that $J(u) \geq 0$ when $\|u\|>1$.

Case 2: $\|u\|>1$.

Note that in this case we have $\phi(\|u\|)=0$, and there exists $i=i(u) \in\{1,2, \ldots, N\}$ such that $\left|\frac{\partial u}{\partial x_{i}}\right|_{p_{i}} \geq \frac{1}{N}$. So,

$$
\begin{aligned}
J(u) & =\sum_{i=1}^{N} \frac{1}{p_{i}}\left|\frac{\partial u}{\partial x_{i}}\right|_{p_{i}}^{p_{i}}-\lambda \frac{1}{q}|u|_{q}^{q} \\
& \geq \frac{1}{p_{i}}\left|\frac{\partial u}{\partial x_{i}}\right|_{p_{i}}^{p_{i}}-\frac{C}{q} \lambda\left|\frac{\partial u}{\partial x_{i}}\right|_{p_{i}}^{q} \\
& =g_{i}\left(\left|\frac{\partial u}{\partial x_{i}}\right|_{p_{i}}\right),
\end{aligned}
$$

where $g_{i}:[1 / N, \infty) \rightarrow \mathbb{R}$ is defined by

$$
g_{i}(t)=\frac{1}{p_{i}} t^{p_{i}}-\frac{C}{q} \lambda t^{q}, \quad i=1, \ldots, N,
$$

which has a global minimum point at $t_{i}=(C \lambda)^{\frac{1}{p_{i}-q}}$ and

$$
g_{i}\left(t_{i}\right)=(C \lambda)^{\frac{p_{i}}{p_{i}-q}}\left(\frac{1}{p_{i}}-\frac{1}{q}\right)<0 .
$$

Observe that $g_{i}(t) \geq 0$ if, and only if, $t \geq\left(\frac{C p_{i}}{q} \lambda\right)^{\frac{1}{p_{i}-q}}$. Hence, to ensure that $\min _{\substack{t \geq \frac{1}{N} \\ 1 \leq i \leq N}} g_{i}(t) \geq 0$, we take $\lambda^{*} \leq \min _{1 \leq i \leq N} \frac{q}{C p_{i} N^{p_{i}-q}}$. Therefore, for each $\lambda \in\left(0, \lambda^{*}\right)$ we have $J(u) \geq 0$ for all $\|u\| \geq 1$. Moreover, we conclude that the functional $J$ is coercive and bounded from below.

Now, we will show that $J$ satisfies the local Palais-Smale condition. For this, we need the following technical result. 
Lemma 5.2. Let $\left(u_{n}\right) \subset D_{0}^{1, \vec{p}}(\Omega)$ be a bounded sequence such that

$$
I\left(u_{n}\right) \rightarrow c \text { and } I^{\prime}\left(u_{n}\right) \rightarrow 0 .
$$

If

$$
\begin{aligned}
c & <\left(\frac{1}{p_{N}}-\frac{1}{p^{*}}\right) S^{p^{*} /\left(p^{*}-p_{N}\right)} \\
& -\left[\frac{\left(\frac{1}{q}-\frac{1}{p_{N}}\right)|\Omega|^{p^{*}-q}}{\left(\frac{1}{p_{N}^{*}}-\frac{1}{p^{*}}\right)}\right]^{p^{*} /\left(p^{*}-q\right)}\left[\left(\frac{q}{p^{*}}\right)^{p^{*} /\left(p^{*}-q\right)}-\left(\frac{q}{p^{*}}\right)^{q /\left(p^{*}-q\right)}\right] \lambda^{p^{*} /\left(p^{*}-q\right)}
\end{aligned}
$$

hold, then there exists $\lambda^{*}>0$ such that, for all $\lambda \in\left(0, \lambda^{*}\right)$, we have that, up to a subsequence, $\left(u_{n}\right)$ is strongly convergent in $D_{0}^{1, \vec{p}}(\Omega)$.

Proof: Using a version of Lions's concentration compactness-principle (see [15, Corollary 1 of Lemma 5$]$ ), we obtain at most a countable index set $\Lambda$, sequences $\left(x_{j}\right) \subset \bar{\Omega},\left(b_{j}\right),\left(a_{j}\right) \subset(0, \infty)$, such that

$\sum_{i=1}^{N}\left|\frac{\partial u_{n}}{\partial x_{i}}\right|^{p_{i}} \rightarrow \sum_{i=1}^{N}\left|\frac{\partial u}{\partial x_{i}}\right|^{p_{i}}+\mu$ and $\left|u_{n}\right|^{p^{*}} \rightarrow|u|^{*^{*}}+\nu \quad$ (weak*-sense of measures),

where

$$
\mu \geq \sum_{j \in \Lambda} b_{j} \delta_{x_{j}}, \quad \nu=\sum_{j \in \Lambda} a_{j} \delta_{x_{j}}, \quad S a_{j}^{p_{N} / p^{*}} \leq b_{j},
$$

for all $j \in \Lambda$ and $\delta_{x_{j}}$ is the Dirac mass at $x_{j} \in \bar{\Omega}$.

Now, for every $\varrho>0$, we set $\psi_{\varrho}(x):=\psi\left(\left(x-x_{j}\right) / \varrho\right)$ where $\psi \in C_{0}^{\infty}\left(\mathbb{R}^{N},[0,1]\right)$ is such that $\psi \equiv 1$ on $B_{1}(0), \psi \equiv 0$ on $\mathbb{R}^{N} \backslash B_{2}(0)$ and $|\nabla \psi|_{\infty} \leq 2$. Since $\left(\psi_{\varrho} u_{n}\right)$ is bounded, $I^{\prime}\left(u_{n}\right)\left(\psi_{\varrho} u_{n}\right) \rightarrow 0$, that is,

$$
\begin{aligned}
\sum_{i=1}^{N} \int_{\Omega} \psi_{\varrho}\left|\frac{\partial u_{n}}{\partial x_{i}}\right|^{p_{i}} d x & =-\sum_{i=1}^{N} \int_{\Omega}\left|\frac{\partial u_{n}}{\partial x_{i}}\right|^{p_{i}-2} \frac{\partial u_{n}}{\partial x_{i}} \frac{\partial \psi_{\varrho}}{\partial x_{i}} d x \\
& +\lambda \int_{\Omega}\left|u_{n}\right|^{q} \psi_{\varrho} d x+\int_{\Omega} \psi_{\varrho}\left|u_{n}\right|^{p^{*}} d x+o_{n}(1) .
\end{aligned}
$$

Arguing as [4], we can prove that

$$
\lim _{\varrho \rightarrow 0}\left[\lim _{n \rightarrow \infty} \sum_{i=1}^{N} \int_{\Omega}\left|\frac{\partial u_{n}}{\partial x_{i}}\right|^{p_{i}-2} \frac{\partial u_{n}}{\partial x_{i}} \frac{\partial \psi_{\varrho}}{\partial x_{i}} d x\right]=0 .
$$

Moreover, since $u_{n} \rightarrow u$ in $L^{q}(\Omega)$ and $\psi_{\varrho}$ has compact support, we can let $n \rightarrow \infty$ in the above expression to obtain

$$
\int_{\Omega} \psi_{\varrho} \mathrm{d} \nu \geq \int_{\Omega} \psi_{\varrho} \mathrm{d} \mu
$$


Letting $\varrho \rightarrow 0$ we conclude that $a_{j} \geq b_{j}$. Since $S a_{j}^{p_{N} / p^{*}} \leq b_{j}$ we have that

$$
S^{p^{*} /\left(p *-p_{N}\right)} \leq a_{j} .
$$

Now we shall prove that the above expression cannot occur, and therefore the set $\Lambda$ is empty. Indeed, arguing by contradiction, let us suppose that the inequality (5.6) holds for some $j \in \Lambda$. Thus,

$$
c=I\left(u_{n}\right)-\frac{1}{p_{N}} I^{\prime}\left(u_{n}\right) u_{n}+o_{n}(1) .
$$

Hence

$$
\left(\frac{1}{p_{N}}-\frac{1}{p^{*}}\right) \int_{\Omega} \psi_{\varrho}\left|u_{n}\right|^{p^{*}} d x-\lambda\left(\frac{1}{q}-\frac{1}{p_{N}}\right) \int_{\Omega}\left|u_{n}\right|^{q} d x \leq c+o_{n}(1) .
$$

Letting $n \rightarrow \infty$, we get

$$
\begin{aligned}
\left(\frac{1}{p_{N}}-\frac{1}{p^{*}}\right) \int_{\Omega}|u|^{p^{*}} d x & +\left(\frac{1}{p_{N}}-\frac{1}{p^{*}}\right) S^{p^{*} /\left(p^{*}-p_{N}\right)} \\
& -\lambda\left(\frac{1}{q}-\frac{1}{p_{N}}\right) \int_{\Omega}|u|^{q} d x \leq c .
\end{aligned}
$$

By Holder's inequality

$$
\begin{aligned}
\left(\frac{1}{p_{N}}-\frac{1}{p^{*}}\right) \int_{\Omega}|u|^{p^{*}} d x & +\left(\frac{1}{p_{N}}-\frac{1}{p^{*}}\right) S^{p^{*} /\left(p^{*}-p_{N}\right)} \\
& -\lambda\left(\frac{1}{q}-\frac{1}{p_{N}}\right)|\Omega|^{\left(p^{*}-q\right) / p^{*}}\left(\int_{\Omega}|u|^{p^{*}} d x\right)^{q / p^{*}} \leq c .
\end{aligned}
$$

Let

$$
f(t)=\left(\frac{1}{p_{N}}-\frac{1}{p^{*}}\right) t^{p^{*}}-\lambda\left(\frac{1}{q}-\frac{1}{p_{N}}\right)|\Omega|^{\frac{p^{*}-q}{p^{*}}} t^{q} .
$$

This function attains its absolute minimum, for $t>0$, at the point

$$
t_{0}=\left[\frac{q \lambda\left(\frac{1}{q}-\frac{1}{p_{N}}\right)|\Omega|^{\frac{p^{*}-q}{p^{*}}}}{p^{*}\left(\frac{1}{p_{N}}-\frac{1}{p^{*}}\right)}\right]^{1 /\left(p^{*}-q\right)} .
$$

Thus, we conclude that

$$
\begin{aligned}
& \left(\frac{1}{p_{N}}-\frac{1}{p^{*}}\right) S^{\left(p^{*}-p_{N}\right) / p} \\
- & {\left[\frac{\left(\frac{1}{q}-\frac{1}{p_{N}}\right)|\Omega|^{\frac{p^{*}-q}{p^{*}}}}{\left(\frac{1}{p_{N}}-\frac{1}{p^{*}}\right)}\right]^{p^{*} /\left(p^{*}-q\right)}\left[\left(\frac{q}{p^{*}}\right)^{p^{*} /\left(p^{*}-q\right)}-\left(\frac{q}{p^{*}}\right)^{q /\left(p^{*}-q\right)}\right] \lambda^{p^{*} /\left(p^{*}-q\right)} } \\
\leq & c .
\end{aligned}
$$


But this is a contradiction. Thus $\Lambda$ is empty and it follows that $u_{n} \rightarrow u$ in $L^{p^{*}}(\Omega)$. Arguing as in the proof of Lemma 4.2, we find

$$
\left\|u_{n}-u\right\|=o_{n}(1)
$$

By the Lemma 5.2 we conclude, for $\lambda>0$ sufficiently small, that

$$
\begin{aligned}
& \left(\frac{1}{p_{N}}-\frac{1}{p^{*}}\right) S^{\left(p^{*}-p_{N}\right) / p} \\
- & {\left[\frac{\left(\frac{1}{q}-\frac{1}{p_{N}}\right)|\Omega|^{\frac{p^{*}-q}{p^{*}}}}{\left(\frac{1}{p_{N}}-\frac{1}{p^{*}}\right)}\right]^{p^{*} /\left(p^{*}-q\right)}\left[\left(\frac{q}{p^{*}}\right)^{p^{*} /\left(p^{*}-q\right)}-\left(\frac{q}{p^{*}}\right)^{q /\left(p^{*}-q\right)}\right] \lambda^{p^{*} /\left(p^{*}-q\right)}>0 }
\end{aligned}
$$

and, hence, if $\left(u_{n}\right)$ is a sequence bounded such that $I\left(u_{n}\right) \rightarrow c, I^{\prime}\left(u_{n}\right) \rightarrow 0$ with $c<0$, then $\left(u_{n}\right)$ has a subsequence convergent.

Lemma 5.3. If $J(u)<0$, then $\|u\|<R_{0}(\lambda)$, for all $i \in\{1, \ldots, N\}$ and $J(v)=I(v)$, for all $v$ in a small enough neighborhood of $u$. Moreover, $J$ verifies a local Palais-Smale condition for $c<0$.

Proof: Since $\lambda \in\left(0, \lambda^{*}\right)$ then $J(u) \geq 0$ whenever $\|u\| \geq 1$. Hence, if $J(u)<0$ we have $\|u\|<1$ and consequently $\bar{g}(\|u\|) \leq J(u)<0$. Therefore, $\|u\|<R_{0}(\lambda)$ and $J(u)=I(u)$. Moreover, we conclude that $J(v)=I(v)$, for all $\|v-u\|<R_{0}(\lambda)-\|u\|$. Moreover, if $\left(u_{n}\right)$ is a sequence such that $J\left(u_{n}\right) \rightarrow c<0$ and $J^{\prime}\left(u_{n}\right) \rightarrow 0$, for $n$ sufficiently large, $I\left(u_{n}\right)=J\left(u_{n}\right) \rightarrow c<0$ and $I^{\prime}\left(u_{n}\right)=J^{\prime}\left(u_{n}\right) \rightarrow 0$. Since $J$ is coercive, we get that $\left(u_{n}\right)$ is bounded in $D_{0}^{1, \vec{p}}(\Omega)$. From Lemma 5.2, for $\lambda$ sufficiently small,

$$
\begin{aligned}
c & <\left(\frac{1}{p_{N}}-\frac{1}{p^{*}}\right) S^{\left(p^{*}-p_{N}\right) / p} \\
& -\left[\frac{\left(\frac{1}{q}-\frac{1}{p_{N}}\right)|\Omega|^{\frac{p^{*}-q}{p^{*}}}}{\left(\frac{1}{p_{N}}-\frac{1}{p^{*}}\right)}\right]^{p^{*} /\left(p^{*}-q\right)}\left[\left(\frac{q}{p^{*}}\right)^{p^{*} /\left(p^{*}-q\right)}-\left(\frac{q}{p^{*}}\right)^{q /\left(p^{*}-q\right)}\right] \lambda^{p^{*} /\left(p^{*}-q\right)}
\end{aligned}
$$

and, hence, up to a subsequence, $\left(u_{n}\right)$ is strongly convergent in $D_{0}^{1, \vec{p}}(\Omega)$.

Now, we will construct an appropriate mini-max sequence of negative critical values for the functional $J$. Thus, for each real number $\epsilon$, we consider the set

$$
J^{-\epsilon}=\left\{u \in D_{0}^{1, \vec{p}}(\Omega): J(u) \leq-\epsilon\right\} \in \mathfrak{A} .
$$

Lemma 5.4. Given $k \in \mathbb{N}$, there exists $\epsilon=\epsilon(k)>0$ such that

$$
\gamma\left(J^{-\epsilon}\right) \geq k
$$


Proof: Since

$$
J(u) \leq \frac{1}{p_{1}} \sum_{i=1}^{N}\left|\frac{\partial u}{\partial x_{i}}\right|_{p_{i}}^{p_{i}}-\lambda \frac{1}{q} C(k)\|u\|^{q},
$$

we can argue as proof of Theorem 1.1 and conclude, there exists $\epsilon=\epsilon(k)$ such that

$$
\gamma\left(J^{-\epsilon}\right) \geq k
$$

We define now, for each $k \in \mathbb{N}$, the sets

$$
\begin{aligned}
\Gamma_{k} & =\left\{C \subset D_{0}^{1, \vec{p}}(\Omega): C \in \mathfrak{A} \text { and } \gamma(C) \geq k\right\}, \\
K_{c} & =\left\{u \in D_{0}^{1, \vec{p}}(\Omega): J^{\prime}(u)=0 \text { and } J(u)=c\right\}
\end{aligned}
$$

and the number

$$
c_{k}=\inf _{C \in \Gamma_{k}} \sup _{u \in C} J(u) .
$$

Lemma 5.5. Given $k \in \mathbb{N}$, the number $c_{k}$ is negative.

Proof: It is sufficient to use Lemma 5.4 and to argument as in [4].

The next Lemma allows us to prove the existence of critical points of $J$. The proof is very similar to that in [4], we omit it here.

Lemma 5.6. If $c=c_{k}=c_{k+1}=\ldots=c_{k+r}$ for some $r \in \mathbb{N}$, then there exists $\lambda^{*}>0$ such that

$$
\gamma\left(K_{c}\right) \geq r+1
$$

for $\lambda \in\left(0, \lambda^{*}\right)$.

\subsection{Proof of Theorem 1.3}

If $-\infty<c_{1}<c_{2}<\ldots<c_{k}<\ldots<0$ with $c_{i} \neq c_{j}$, since each $c_{k}$ is critical value of $J$, the we obtain infinitely many critical points of $J$ and, hence problem $\left(P 2_{\lambda}\right)$ has infinitely many solutions.

On the other hand, if there are two constants $c_{k}=c_{k+r}$, then $c=c_{k}=$ $c_{k+1}=\ldots=c_{k+r}$ and from Lemma 5.6, there exists $\lambda^{*}>0$ such that

$$
\gamma\left(K_{c}\right) \geq r+1 \geq 2
$$

for all $\lambda \in\left(0, \lambda^{*}\right)$. From Proposition $3.2, K_{c}$ has infinitely many points, that is, problem $\left(P 2_{\lambda}\right)$ has infinitely many solutions. 


\section{References}

[1] C. O. Alves and A. El Hamidi, Existence of solution for a anisotropic equation with critical exponent, Differential Integral Equations, 21, (2008), no. 1-2, 2540.

[2] A. Ambrosetti and P. H. Rabinowitz, Dual variational methods in critical point theory and apllications, J. Functional Analysis, 14, (1973), 349-381.

[3] S. Antontsev, J. I Díaz and S. Shmarev, Energy methods for free boundary problems: applications to nonlinear PDEs and fluid mechanics, In: Progress in Nonlinear Differential Equations and their Applications, 48, Birkhäse, Boston (2002).

[4] J. García-Azorero and I. Peral, Multiplicity of solutions for elliptic problems with critical exponent or with a nonsymmetric term, Trans. Amer. Math. Soc., 323, (1991), 877-895.

[5] J. Bear, Dynamics of Fluids in Porous Media, American Elsevier, New York (1972).

[6] V. Benci, On critical points theory for indefinite functionals in the presence of symmetric, Trans. Amer. Math. Soc. 274 (1982) 533-572.

[7] M. Bendahmane and K. H. Karlsen, Renormalized solutions of an anisotropic reaction-diffusion-advection system with $L^{1}$ data, Commun. Pure Appl. Anal. $5(4),(2006)$ 733-762.

[8] M. Bendahmane, M. Langlais and M. Saad, On some anisotropic reactiondiffusion systems with $L^{1}$-data modeling the propagation of an epidemic disease., Nonlinear Anal. 54(4), (2003) 617-636.

[9] A. Di Castro, M. Pérez-Llanos and J. M. Urbano, Limits of anisotropic and degenerate elliptic problems, Comm. Pure Appl. Anal., 11, (2012), 1217-1229.

[10] A. Di Castro, Existence and Regularity Results for Anisotropic Elliptic Problems, Adv. Nonl. Studies, 9, (2009), 367-293.

[11] A. Di Castro and E. Montefusco, Nonlinear eigenvalues for anisotropic quasilinear degenerate elliptic equations, Nonl. Analysis, 70, (2009), 40934105.

[12] A. Castro, Metodos variacionales y analisi functional no linear, X Coloquio colombiano de Matematicas, 1980.

[13] D. G. Costa, Topicos em analise não linear e aplicações as equações diferenciais, Escola Latino-Americano de Matematica, 1986. 
[14] G. M Figueiredo and J. R. Santos Júnior, Multiplicity of solutions for a Kirchhoff equation with subcritical or critical growth, Differential and Integral Equations, 25, (2012), 853-868.

[15] A. El Hamidi and J.M. Rakotoson, Extremal functions for the anisotropic Sobolev inequalities, Ann. I. H. Poincaré, 24, (2007), 741-756.

[16] A. El Hamidi and J.M. Rakotoson, Compactness and quasilinear problems with critical exponent, Diff. Int. Equation, 18, (2005), 1201-1220.

[17] I. Fragala, F. Gazzola, and B. Kawohl, Existence and nonexistence results for anisotropic quasilinear elliptic quations, Ann. Inst. H. Poincaré Anal. Non Linéaire, 21, (2004), 715-734.

[18] M. A. Kranolseskii, Topological methods in the theory of nonlinear integral equations. MacMillan, New York, 1964.

[19] D. C Clark, A variant of the Lusternik-Schnirelman theory, Indiana Univ. Math., J. 22, (1972), 65-74.

[20] S.N. Kruzhkov and I.M. Kolodii, On the theory of embedding of anisotropic Sobolev spaces, Russian Math. Surveys 38, (1983), 188-189.

[21] S.M. Nikol'skii, On imbedding, continuation and approximation theorems for differentiable functions of several variables, Russian Math. Surv. 16, (1961), 55-104.

[22] J. Rákosnik, Some remarks to anisotropic Sobolev spaces I, Beiträge zur Analysis, 13, (1979), 55-69.

[23] J. Rákosnik, Some remarks to anisotropic Sobolev spaces II, Beiträge zur Analysis, 13, (1981), 127-140.

[24] M. Troisi, Teoremi di inclusione per spazi di Sobolev non isotropi, Ricerche Mat., 18, (1969), 3-24.

[25] J. Weickert, Anisotropic diffusion in image processing, European Consortium for Mathematics in Industry, B. G. Teubner, Stuttgart, 1998.

[26] J. Vétois, The blow-up of critical anisotropic equations with critical directions, Nonlinear Differ. Equ. Appl. 18, (2011), 173-197.

[27] J. Vétois, Existence and regularity for critical anisotropic equations with critical directions, Adv. Differential Equations 16, (2011), 61-83. 